\title{
De l'activité DE simulation à l'activité EN simulation : simuler pour stimuler
}

Marc-Éric Bobillier Chaumon, Sabrina Rouat, Elsa Laneyrie et Bruno Cuvillier

\section{OpenEdition}

\section{Journals}

Édition électronique

URL : http://journals.openedition.org/activites/3136

DOI : $10.4000 /$ activites.3136

ISSN : 1765-2723

Éditeur

ARPACT - Association Recherches et Pratiques sur les ACTivités

Référence électronique

Marc-Éric Bobillier Chaumon, Sabrina Rouat, Elsa Laneyrie et Bruno Cuvillier, « De l'activité DE simulation à l'activité EN simulation : simuler pour stimuler», Activités [En ligne], 15-1 | 2018, mis en ligne le 15 avril 2018, consulté le 19 avril 2019. URL : http://journals.openedition.org/activites/3136 ; DOI : 10.4000/activites.3136

Ce document a été généré automatiquement le 19 avril 2019

Licence Creative Commons

Activités est mis à disposition selon les termes de la licence Creative Commons Attribution - Pas d'Utilisation Commerciale - Pas de Modification 4.0 International. 


\title{
De l'activité DE simulation à l'activité EN simulation: simuler pour stimuler
}

\author{
Marc-Éric Bobillier Chaumon, Sabrina Rouat, Elsa Laneyrie et Bruno \\ Cuvillier
}

\section{NOTE DE L'ÉDITEUR}

Article soumis le 14/11/2017, accepté le 28/02/2018

\begin{abstract}
« Aujourd'hui l'abstraction n'est plus celle de la carte, du double, du miroir ou du concept. La simulation n'est plus celle d'un territoire, d'un être référentiel, d'une substance. Elle est la génération par les modèles d'un réel sans origine ni réalité : hyperréel. Le territoire ne précède plus la carte, ni ne lui survit». Jean Baudrillard (1981) dans « Simulacres et simulation »
\end{abstract}

\section{Introduction}

Les changements nombreux et réguliers auxquels sont confrontés les salariés, les innovations qui se déploient de façon plus ou moins brutale dans nos différents systèmes de vie (domestique, professionnelle, sociale...) nous rappellent combien la conception et la transformation des situations de vie doivent être pensées et soutenues par des démarches appropriées. L'analyse de l'activité ainsi que la conception participative (centrée utilisateur et activité) ont démontré leur utilité pour rendre compte d'une part, des conditions d'exercice de l'activité, et pour développer d'autre part des solutions 
intégrant les caractéristiques des futurs usagers et les spécificités de leurs pratiques. La simulation de l'activité serait le troisième pilier de ce dispositif d'accompagnement des transformations.

Mais que recouvre précisément la simulation de l'activité ? Quelles sont les méthodes associées? Que visent-elles et sur quels objets et activités interviennent-elles ? Pour quels bénéfices, mais aussi avec quelles limites? D’ailleurs doit-on parler de simulation De l'activité ou de simulation Des activités, tant le champ d'application et le spectre des usages paraissent importants? Quels sont aussi les différentes formes et types de simulations possibles?

3 Sur la base des différents retours d'expérience de la journée d'étude sur la Simulation organisée par la revue Activités - nous souhaitons proposer une synthèse critique qui rende compte des différents apports et contributions des méthodes de simulation. Nous cherchons plus particulièrement à définir une taxonomie fonctionnelle et diachronique de ces différentes techniques de simulation, dans l'optique de dégager leurs intérêts et limites respectifs. Nous verrons que selon les finalités et orientations temporelles (simuler une activité à venir, en devenir ou passée), il est également possible de mobiliser d'autres méthodes, qui sont traditionnellement utilisées comme des démarches d'analyse et de restitution de l'activité. L'ambition est alors de montrer en quoi et pourquoi ces différentes approches peuvent être considérées comme des cadres d'intervention permettant de simuler pour agir sur l'activité, dans une visée à la fois réflexive, constructive et transformatrice du sujet de son activité et de la situation.

\section{Les visées de la simulation : objets et finalités}

Selon l'étymologie du terme simulation (apparue à la fin du XII ${ }^{\text {ème }}$ siècle), celle-ci serait une : «action de feindre, de faire paraitre réelle une chose qui ne l'est pas » (Thomas de Kent, 2003). Cette définition introduit une certaine liberté laissée aux individus ; soit celle de jouer avec l'activité, du moins dans sa manifestation réalisée. Les différents travaux présentés lors de la journée d'étude sur les nouvelles figures de la simulation ont cependant montré que la simulation pouvait aussi s'ouvrir sur d'autres usages et finalités que la simple « contrefaçon » ou imitation d'une activité.

Elle pouvait ainsi porter sur des retours d'expériences (praxiques, émotionnelles, cognitives, subjectives) à partir desquelles les personnes allaient pouvoir (s')éprouver, élaborer - individuellement et/ou collectivement - et développer les ressources idoines pour se transformer ou transformer les systèmes d'activité dans lesquelles les professionnels opèrent. Dans ce cas, il ne s'agit donc plus seulement de feindre ou de jouer une activité, comme l'acception première du terme "simulation" l'entend, mais d'être en capacité de se confronter à une réalité, la sienne propre ou celle d'un autre, afin de revivre ou de ressentir différemment les choses. C'est ce rapport distancié à l'activité qu'introduit cette autre perspective de la simulation. Elle permet à l'individu de se percevoir différemment et ce faisant, d'envisager aussi différemment les conditions d'accomplissement de l'activité et de son propre accomplissement. Par cette voie de réflexion renouvelée de la simulation et de son usage, il nous semble donc possible de mobiliser des méthodes d'analyse de l'activité qui, si à première vue paraissent éloignées de la définition originelle de la simulation, peuvent néanmoins offrir un cadre propice à 
la réflexivité sur l'activité et à la transformation des pratiques, des situations et du sujet lui-même.

Dans les sections qui vont suivre, nous présenterons les différentes visées de la simulation portant sur la conception, la correction/transformation et enfin l'étayage des activités.

\subsection{Simuler pour concevoir}

7 Dans son acception générale, la simulation d'activité a pour ambition de mettre en œuvre une démarche pour accompagner les projets de transformation ou de conception en plaçant l'activité et les individus au cœur de l'intervention. Elle consiste à se déporter imaginairement hors du réel en "faisant comme si ", c'est-à-dire en étant à la fois acteur et spectateur de l'activité qui se fait et/ou qui se fera. Elle permet à la fois « une exploration du champ des possibles et participe aussi à une réduction d'incertitude » (Daniellou, 2007, p. 9) dans la mesure où elle cherche à articuler les attentes diverses et parfois contradictoires des différentes parties prenantes du projet. Elle vise enfin, par l'exploration des solutions possibles, à concilier le développement de l'activité des individus avec la performance des organisations. Mais la simulation de l'activité est plus qu'une « simple » activité de simulation. Elle ne cherche pas seulement à faire tester une situation future-probable afin d'en évaluer la pertinence et l'opportunité auprès d'usagers finaux. Elle ambitionne aussi de coconstruire les conditions de réalisation de cette nouvelle activité, pour mieux y inscrire le projet.

8 De plus, en privilégiant la confrontation à une forme possible ou plausible du réel, la simulation permet d'expérimenter les limites probables du dispositif ainsi que ses propres limites. L'individu peut ainsi mesurer les incidences qu'aura le dispositif dans le déroulement ou le renouvellement de son activité : ce qu'il permet, autorise, force ou empêche de faire, mais aussi ce qu'il permet de mieux faire, moins bien ou différemment. Ces questions d'efficience et de sens de l'activité s'avèrent d'ailleurs cruciales dans l'acceptation de toute innovation (Bobillier Chaumon, \& Clot, 2016). De même que les individus auront l'opportunité de vérifier quelles sont les marges de manœuvre dont ils disposent, pour non seulement accorder le futur système à leur manière de fonctionner, mais aussi pour repenser plus globalement la situation dans laquelle s'intégrera in fine le dispositif. Ici la simulation contribue à ajuster le couplage individu-situation afin que l'un et l'autre s'accordent au mieux durablement.

C'est donc une manière - pour les individus et les collectifs - de (re)donner du sens au projet de changement en l'inscrivant dans un cadre connu et signifiant, et en engageant/ amorçant un processus d'appropriation de l'innovation. C'est parce que la simulation donne justement la possibilité de «jouer» avec les objets, de s'arranger avec les modalités et les contours de la future activité, qu'ils deviennent acceptables pour les individus dans l'activité qui se construit (Bobillier Chaumon, 2016). Comme le rappelle d'ailleurs Daniellou (2007), l'objectif de la simulation n'est pas d'effectuer une prescription concernant une bonne façon de réaliser la tâche, mais plutôt de comprendre comment l'activité future probable peut se développer et si ces formes possibles sont acceptables pour les différents protagonistes, non seulement vis-à-vis de la situation qu'ils vivent actuellement, mais aussi par rapport à celle à laquelle ils seront confrontés dans un avenir plus ou moins proche. Notre contribution s'inspire des apports respectifs de Vygotski $(1928$; 1932) et de Winnicott (1971) pour qui le «jeu», permet de libérer les 
potentialités de créativité des sujets, dans un mouvement de trouver/créer qui assure la maîtrise du rapport du sujet à son milieu.

\subsection{Simuler pour corriger et transformer}

10 Sur un autre registre, la simulation peut aussi viser à repenser la situation de travail existante pour améliorer les modalités d'actions individuelles et collectives. De nouvelles perspectives pour l'activité - qui jusque-là étaient impensées, refoulées, empêchées peuvent dès lors être ouvertes par la confrontation à ses propres limites. Dans ce cadre, la méthode de simulation constitue un lieu propice à la mobilisation de la subjectivité et de la créativité des individus. Un espace au sein duquel les capacités d'innovation et d'improvisation qui caractérisent le réel de l'activité (Clot, 2008) pourront aussi être portées à la (re)connaissance des différentes parties prenantes de l'activité (concepteur, décideur, manager, prescripteur) : la simulation fonctionne dès lors comme un moyen pour « ouvrir l'espace de conception » et nourrir l'interaction (discussion et négociation) entre les acteurs engagés dans la conception autour de différentes alternatives possibles » (Salembier, \& Pavard, 2004, p. 95).

\subsection{Simuler pour étayer}

11 Sur un autre registre encore, la simulation peut conduire à la mise en visibilité d'une activité plus intime, plus subjective du salarié au travail. Non seulement ses manières d'être et de faire, mais aussi ses doutes et ses craintes, ses fragilités et ses souffrances... peuvent s'y dévoiler, s'y exprimer et pourront dès lors y être discutées. Cette expérience émotionnelle d'une activité - encore - vivante (et pas seulement vécue) pourra aussi être travaillée lors de la simulation. Les éprouvés persistants, déplaisants pourront dès lors être «contenus » à travers le travail d'élaboration que suppose la simulation (par des dispositifs comme le psychodrame ou encore le théâtre forum).

En définitive, la simulation conduit donc à une mise en perspective de l'activité, à une prise de recul sur celle-ci. Elle donne à l'individu la possibilité soit de vivre ou de revivre des situations (passées ou contemporaines, réalisées ou non réalisées), soit d'expérimenter des situations/projets à venir pour mieux les penser (ou les repenser) et agir sur eux. Ce retour sur le passé est parfois nécessaire pour s'autoriser à se projeter dans le futur. Il y a donc des possibilités de développement et d'ajustement qui touchent le sujet, mais également l'activité dans laquelle il s'inscrit. En d'autres termes, la simulation ne se cantonne pas à une simple répétition ou restitution de l'activité visée. C'est bien la confrontation à cette activité, dans ses possibilités et ses impossibilités, qui va provoquer des perspectives d'évolution et d'innovation, qui va susciter de nouveaux horizons, qui va permettre d'imaginer d'autres façons d'être, d'agir et de collaborer dans la situation, et qui peut conduire aussi à proposer des aménagements à l'activité et au projet. Il faut ici entendre provoquer aux deux sens du terme: provoquer pour déclencher une réaction, mais également provoquer pour heurter l'individu dans ses acquis et certitudes, pour le faire bouger et réagir dans ses pratiques et ses regards. 


\section{Quels critères retenir pour organiser les formes de simulation?}

13 On le voit donc, la simulation peut revêtir différentes formes et avoir différentes vocations. Elle peut ainsi chercher à :

- Développer l'expérience, les compétences et/ou l'expertise des personnes en les confrontant à des situations simulées et contrôlées : Simulation de l'activité sur des environnements immersifs et persistants comme les serious-game, système à réalité augmentée ou virtuelle,

- Concevoir un projet innovant (technologique, socio-organisationnel, collaboratif...) en impliquant les usagers finaux et les différents protagonistes du projet par confrontation au devenir possible de leur activité : démarche de simulation de l'activité future-probable, living-lab...

- Travailler sur des éprouvés émotionnels générés par des épisodes professionnels traumatisants dans une visée thérapeutique et cathartique (simulation par psychodrame, théâtre forum, debriefing)...

Développer des ressources pour l'activité en cours et pour son développement, par une mise en perspective de ses propres pratiques : méthodes du sosie, de l'auto-confrontation, de l'objet technique....

Les méthodes de simulation peuvent se caractériser aussi en fonction de critères plus opérationnels et systémiques qui ont trait aux :

- Ambitions de la simulation: s'agit-il d'accompagner et de nourrir la conception, de mobiliser et d'impliquer les acteurs (conception participative), de développer des ressources individuelles et collectives, de corriger des situations existantes, d'ajuster les situations à venir, de rendre plus visible et lisible l'activité, de favoriser la qualité du travail, de coconstruire les conditions d'acceptation des systèmes innovants ? ...

- Objets simulés : ceux-ci peuvent avoir trait à l'activité socio-domestique ou professionnelle, aux compétences, à l'expérience, aux usages, aux collectifs de travail, aux éprouvés subjectifs, sur l'organisation et les espaces de travail, sur la technologie...

- Méthodes déployées: qui peuvent relever de démarches dédiées spécifiquement à la simulation (scénarisation, mise en situation...), mais qui peuvent aussi s'appuyer sur des outils plus classiques d'analyse de l'activité (comme les méthodes d'auto-confrontation, de sosie...).

- Cadres de la situation dans laquelle la simulation se déploie : est-ce un espace réel, recrée/ artificiel ou imaginaire? Est-ce à l'échelle d'une situation, d'un système d'activité ou d'une organisation-institution?

- Rôles de l'intervenant: est-il celui qui initie, anime, accompagne la simulation? Est-il accompagnant, médiateur, observateur, prescripteur, contributeur? Quelles sont les marges de manœuvre dont dispose cet intervenant, compte tenu de la situation de simulation?

- Destinataires à qui s'adresse l'action : il peut s'agir de destinataires directs ou indirects, un sujet lui-même, mais aussi son collectif d'appartenance, les partenaires de l'activité, les concepteurs, les décideurs/managers, les financeurs.

- Distances avec l'objet de la simulation: la situation qui fait l'objet de la simulation, met l'individu en situation d'évocation soit d'une situation vécue personnellement exposant l'individu au contact de son vécu réel (simulation rejouée de façon intrinsèque); soit d'une situation vécue par un ou par d'autres ou encore par un outil (simulation rejouée de façon extrinsèque), un objet technique, informatique ou computationnel. 
- Perspectives temporelles dans lesquelles la simulation s'inscrit: la simulation de l'activité est-elle tournée plutôt vers le passé, le présent ou un futur probable?

\section{Les différents types de simulation}

Selon les différents critères évoqués supra, il est possible de dégager quatre grandes orientations de la simulation pouvant être représentées sur une trajectoire temporelle (Cf. figure 1). Cette mise en perspective diachronique de la simulation nous est apparue centrale afin de rendre compte des différents types de simulation et ainsi envisager leur contribution dans le développement de l'activité des individus. À ce titre, les démarches de simulation peuvent être tournées vers :

i. Une activité passée (terminée), c'est une situation déjà réalisée qui est simulée afin de guider et d'alimenter, par des retours d'expérience, la (re)conception $\rightarrow$ simulation rétrospective.

ii. Une activité vivante : c'est une simulation qui porte sur des éprouvés émotionnels persistants que l'on souhaite travailler $\rightarrow$ simulation projective.

iii. Une activité qui se fait (en devenir) : c'est une situation plus actuelle qu'on souhaite améliorer ou corriger par la simulation $\rightarrow$ simulation réflexive (ou constructive).

iv. Une activité qui peut se faire (à venir): c'est une situation future-probable qu'on souhaite implanter et tester $\rightarrow$ c'est la simulation prospective (ou prédictive).

17 La simulation apparaît à la fois protéiforme, plurielle et inscrite dans des temporalités multiples. Ces orientations peuvent aussi être déclinées dans un graphe selon deux dimensions : la première (ordonnée) représente l'activité dans sa perspective temporelle (comme évoquée supra), la seconde (abscisse) distingue l'activité réalisée de celle non réalisée. Le réel de l'activité et l'activité réelle ne se recoupent effectivement pas. La simulation peut donc porter sur les activités qui se font ou qui se sont faites (activité réelle), mais aussi sur celles qui n'existent formellement pas encore, ou bien encore qui ne peuvent se faire ; comme les activités suspendues, contrariées ou empêchées, voire les contre-activités (i.e. le réel de l'activité selon Clot, 2008).

Figure 1. Les types de simulation.

Figure 1. Simulation types

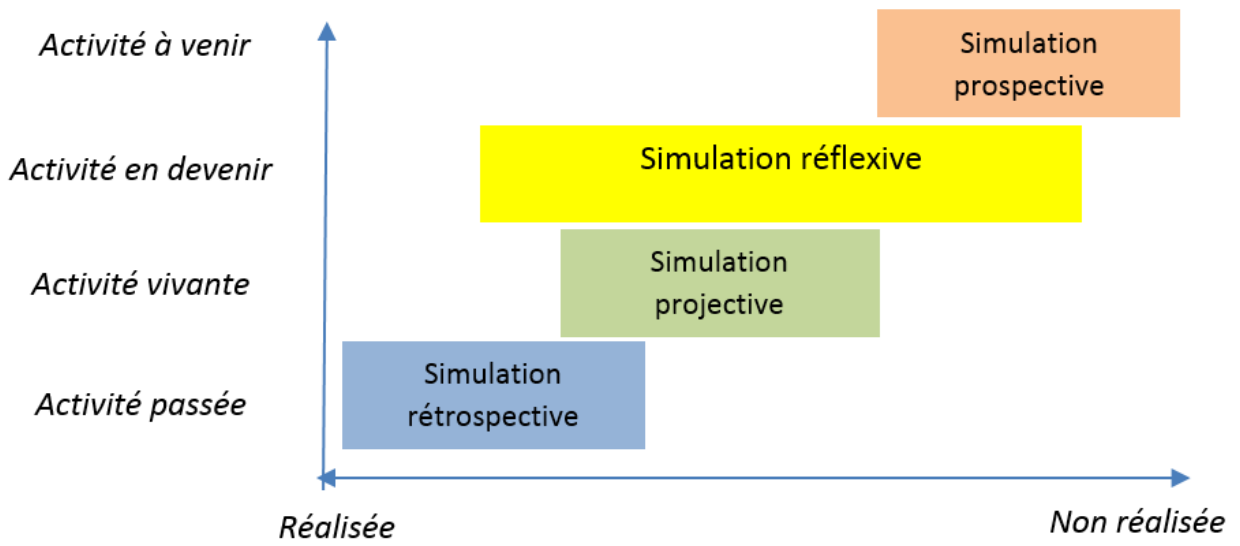

Les sous-parties qui suivent visent à présenter, pour chacun de ces types de simulation, les méthodes susceptibles d'être mobilisées pour instruire la simulation, en précisant leurs différentes attributions (en matière de propriétés, de finalités, de moyens d'action) 
et contributions (en matière d'apports et de limites). Sans viser l'exhaustivité, la présentation tente de saisir la logique propre de chaque type de simulation ainsi que leur articulation possible. Nous verrons aussi, comme déjà évoqué supra, que des méthodes classiques d'analyse et de compréhension de l'activité (comme l'entretien d'explicitation ou l'analyse de la pratique par exemple), peuvent être détournées de leur usage originel pour permettre une mise en situation/scène de l'activité, proche des démarches de simulation.

\subsection{Simulation prospective ou prédictive}

simulation prospective « vise, à partir de la compréhension du travail réel actuel, à "faire jouer" par les personnes concernées le travail futur probable dans les conditions imposées par les nouveaux scénarios de prescriptions proposés par les prescripteurs. " (Barcellini, Van Belleghem, \& Daniellou, 2013, p. 6). L'objectif de cette méthodologie est donc de simuler une activité qui " pourrait se faire », c'est-à-dire qui n'existe pas encore ou qui se trouve bouleversée par l'arrivée d'un nouveau système, environnement ou méthode de travail.

Plus précisément, dans la simulation par scénarisation/ mise en situation (MES), on invite le sujet à jouer son activité dans un nouveau contexte prescriptif. La simulation peut se dérouler de deux façons : soit en grandeur nature c'est-à-dire en utilisant un prototype afin de pouvoir expérimenter le nouvel objet, soit en utilisant un support de simulation réduit, comme par exemple, une maquette dans laquelle les salariés vont utiliser des avatars (Van Belleghem, 2012). Dans les deux cas, les sujets vont commenter l'activité qu'ils "jouent», sur la base de scénarii qui leur sont soumis. Ils décrivent ce qu'ils peuvent faire ou ne plus faire, ou plus difficilement, compte tenu des contraintes rencontrées dans cet environnement simulé. L'utilisation d'un avatar permet de projeter son activité, en la jouant, en incarnant des actions qui sont simulées. Cet objet intermédiaire favorise donc la vraisemblance de la description de l'activité.

L'intérêt de ce type de dispositif est qu'il permet à un individu de confronter les « invariants » qui guident son activité aux nouvelles circonstances dans lesquelles son action pourrait s'inscrire (l'activité simulée à venir). C'est ce travail de réélaboration à la fois de ses pratiques et de ses représentations qui va permettre à l'individu d'apprécier assez concrètement ce que le projet pourrait changer dans l'exercice de son activité, et proposer du coup des ajustements au projet testé.

Outre cette démarche de simulation par scénarisation, d'autres dispositifs méthodologiques permettent aussi au sujet d'expérimenter des situations fictives. C'est le cas du « Magicien d'Oz » qui est une expérience de simulation qui consiste à faire croire aux sujets que le système technique avec lequel ils interagissent est autonome, alors qu'il est en réalité totalement ou partiellement contrôlé/piloté par un humain (Baccino, Bellino, \& Colombi, 2005). Au cours de cette simulation, l'utilisateur pense que la réponse à ses opérations est émise par le dispositif alors qu'elle est en réalité générée par un opérateur caché en direct. L'intérêt de cette démarche est de tester en conditions quasi réelles (au domicile, au travail) un démonstrateur technique ou un prototype qui est en phase de conception. Ces tests en situation s'inscrivent dans une " conception par l'usage " dans la mesure où les retours d'expériences vont permettre l'ajustement du projet technique aux conditions d'utilisation. 
21 Autre démarche de simulation prospective, les Living-labs. Cette méthodologie de recherche en innovation a la particularité de regrouper différents acteurs (publics, privés, citoyens, usagers) qui travaillent de concert - et non en vase clos - afin de concevoir, développer et valider dans des conditions proches du cadre réel de vie, des produits, services et technologies destinés le plus généralement à des personnes fragilisées (malades, âgées...) dans le champ de la santé et de l'autonomie. L'ambition est de recueillir et de confronter les différents points de vue (techniques, sociaux, culturels, économiques) portés par les différentes disciplines, partenaires et acteurs/destinataires du projet innovant (Voilmy, 2017). Les résultats de cette approche participative sont alors mis en scène pour faire "vivre une expérience ", pour proposer un "cadre de jeu » d'une expérience réelle, qui sera déployée en modèle réduit réaliste afin d'en évaluer les répercussions potentielles auprès des diverses parties prenantes du projet : usagers, mais aussi l'ensemble des protagonistes, partenaires et acteurs institutionnels. Chacun pourra ainsi manipuler, expérimenter tout ou partie de la solution afin de s'assurer que celle-ci soit bien pertinente et compatible avec leurs intérêts et attentes, et que surtout les futurs utilisateurs sont prêts à s'en servir.

Le serious game relève aussi d'une méthode de simulation à visée prospective puisqu'elle consiste à projeter des individus, via un avatar, dans des environnements virtuels, le plus souvent dématérialisés ${ }^{1}$, qui se veulent ludiques afin de susciter le plus d'engagement et d'interactivité possible. Ce type de dispositif est utilisé majoritairement dans le cadre de la formation professionnelle. Le joueur doit alors mener différentes missions (i.e. conduire un projet de changement dans une entreprise) selon des scénarii préétablis et en collaborant avec d'autres personnages virtuels (non joueurs) qui apparaissent en cours de jeu. Par cette immersion dans un environnement artificiel qui se veut assez proche de la réalité, le joueur a la possibilité de tester des stratégies, d'explorer des conduites, d'éprouver des scénarii pour en évaluer virtuellement les effets, sans pour autant en subir concrètement les conséquences. Ces expérimentations visent donc à (re)élaborer des compétences et des représentations, potentiellement transférables dans la vraie vie et dans la future situation. Notons aussi que dans le cas du serious gaming, il y a la présence d'un tiers formateur qui joue le rôle d'animateur/médiateur dans le briefing, l'accompagnement et le débriefing. Il oriente et guide la formation et le retour sur expérience, et permet ainsi une réflexivité sur l'expérience vécue (Martin, \& Lhuilier, 2016).

Dans la même veine, mais sur une réalité différente, car elle projette l'individu sur une situation qui n'arrivera pas, il existe le jeu de rôles. Bien que nous soyons dans l'approche prospective, et qu'il invite à explorer des dimensions de l'activité en invitant le participant à se mettre en scène sur une situation virtuelle, nous ne sommes pas ici dans une même réalité. Le travail en question, tel que défini dans le jeu, ne se fera pas, mais permet d'ajuster son attitude et transformer l'activité. En effet, le jeu de rôle est un cadre « ludique » qui permet de simuler une activité dans une situation qui ne se fera pas. Il prend la forme "d'exercices structurés en dynamiques des groupes » (De Visscher, 1979). Il rassemble trois ingrédients de l'apprentissage que sont l'expérience, le groupe et le jeu (Patin, 2005). Ces «situations miniatures» (pour reprendre l'expression de Lewin) favorisent l'implication personnelle dans le processus d'apprentissage. Il propose au professionnel de se mettre en scène lui-même, c'est-à-dire en jouant un rôle fictif. Une modalité supplémentaire peut consister à jouer le rôle d'un autre. Le sujet expérimente alors une capacité à se décentrer pour rentrer dans une attitude centrée sur l'autre. Il 
peut alors oser se montrer, se tromper et recréer une perception de soi-même et de l'autre portée par l'échange entre participants.

Ainsi, dans le serious-game comme dans le jeu de rôle, il apparaît que c'est par la prise de conscience de ses atouts et difficultés, mis en exergue dans le jeu de la situation à travailler ou à expérimenter (virtuellement), que l'individu va être incité à mettre en œuvre de nouvelles pratiques.

Une autre forme de simulation, de type informatique ou "computationnelle», est également développée pour prédire et comprendre les transformations possibles de l'environnement (Salembier, \& Pavard, 2004). Il s'agit plus précisément de reproduire, par une modélisation informatique, les situations et conditions de vie (socio-domestique, professionnelle) ainsi que les profils socio-biographiques des personnes qui y séjournent (sous l'apparence d'agents ou d'avatars). Chaque agent y est ainsi décrit par un ensemble d'attributs qui vont définir un éventail de comportements possibles; ces attributs sont soit prédéfinis, soit générés dynamiquement au cours de la simulation. Cette modélisation informatique des conduites humaines s'appuie sur des analyses d'activité et une compréhension fine des environnements investigués pour être en mesure de les reproduire le plus fidèlement possible (Cf. l'étude de Guibourdenche, Vacherand-Revel, Fréjus, \& Haradji, 2015). Cette représentation artificielle du réel permet alors de tester de manière virtuelle différents scénarii pour déterminer les changements qui pourraient s'opérer dans ces milieux de vie et sur les activités humaines simulées. On peut par exemple citer les travaux de Guibourdenche, Salembier, Poizat, Haradji et Galbat (2015) qui, dans le cadre d'une recherche sur la consommation électrique, testent les effets de différentes conduites (habitudes, choix, pratiques d'une famille) au sein du domicile pour évaluer l'efficience énergétique. Ou encore les travaux de Pallamin, Pavard, Zamberlan et Santos (2010) qui portent sur les interactions sociales professionnelles en univers virtuel avec pour objectif de réaliser des environnements collaboratifs pour la formation et l'activité.

Au final, il apparaît que ces méthodes de simulation prospective, si elles sont toutes tournées vers une activité à venir et si elles mobilisent des démarches de mise en situation dans des conditions réelles, quasi réelles, voire artificielles ou virtuelles, visent bien deux perspectives de transformation différentes: l'une est davantage tournée vers l'ajustement de la situation, du système d'activité et du projet à implanter (cas de la simulation par scénarisation, du living lab, du magicien d'Oz et de la simulation informatique) ; l'autre porte davantage sur l'individu et sa capacité à élargir la gamme de ses possibles pour réinvestir autrement l'activité à venir et développer les ressources idoines (cas du serious game et du jeu de rôles).

\subsection{Simulation constructive ou réflexive}

Cette démarche de simulation constructive ou réflexive porte sur le travail " qui se fait ». Il s'agit de redonner du pouvoir d'agir aux individus dans l'exercice de leur activité. Elle s'insère dans le champ de la professionnalisation et est particulièrement mobilisée par la clinique de l'activité qui se destine à devenir un instrument pour développer la capacité d'agir des individus et des collectifs sur l'environnement et sur eux-mêmes. Elle est un cadre pour que le travail puisse devenir ou redevenir un objet de pensée. Le processus mis en place fait appel à l'analyse d'activité et de confrontation (individuelle et/ou collective) à ses propres pratiques, via des techniques particulières, par une co-analyse des actions 
déployées en situation. Il s'agit plus précisément de permettre aux professionnels d'examiner de manière répétée leur activité et de pouvoir objectiver les tensions du métier sur la base du travail réel, et pas seulement sur la base du travail réalisé. De ce fait, contrairement à la simulation prospective où le sujet cherche à peser sur un projet, la simulation réflexive induit une transformation du sujet lui-même, par l'élaboration de nouvelles ressources. De ce fait elle incarne une aire intermédiaire située en dehors de l'individu et de sa réalité professionnelle qui l'aide à comprendre ses expériences pour mieux appréhender les situations réelles et mieux agir sur elles, par le développement de capacités d'action renouvelées. Ainsi, l'individu peut créer du contexte pour vivre. Ici le réalisé ne recouvre pas le réel de l'activité, qui se retrouve, par différentes techniques, convoqué pour rendre possible son expression.

Cette forme de réflexivité place l'individu dans un processus d'interprétation continu de ses propres réalités : subjectives et intersubjectives. Il ne s'agit pas seulement de faire un retour sur les difficultés et les empêchements de l'activité, mais de prendre du recul sur et surtout avec soi-même à l'aide du dispositif de simulation qui favorise cet exercice de réélaboration. Cette mise en débat par et sur le travail va permettre «aux professionnels impliqués dans l'histoire d'un milieu de développer leur propre expérience, individuelle et collective, pour développer l'histoire du métier lui-même et en faire une ressource pour penser et agir» (Kostulski, Clot, Litim, \& Plateau, 2011, p. 131). Il s'agit d'accompagner les professionnels dans la recherche de nouveaux possibles, "d'éprouver avec eux ce qu'ils pourraient devenir » (Clot, 2008, p. 55), par le regard nouveau et décalé qu'ils portent sur leur activité. Il s'agit donc, par ces techniques de simulation réflexive du réel de l'activité, de mettre au jour et à jour des façons d'agir, des principes de fonctionnement organisationnels, des règles de métier, qui sont plus ou moins adaptés, plus ou moins acceptables dans la recherche d'un travail de qualité. Plusieurs techniques de simulation de l'activité peuvent permettre d'accéder et de révéler cette activité souvent inconsciente, automatisée ou refoulée.

Tout d'abord, la méthode d'instruction au sosie, mise en place par Oddone puis développée par Clot et Faïta (2000), confronte le sujet à lui-même par la médiation de l'activité réglée du sosie. Elle conduit alors à une description très fine de l'activité, où le professionnel va se mettre en scène, va littéralement jouer, simuler les différentes actions de son travail pour permettre à l'autre de le remplacer. La naïveté méthodologique employée dans cette simulation permet d'éviter une description prescriptive des tâches (Miossec, 2011) et d'accéder au rapport à l'activité, aux pairs et à la hiérarchie, à l'organisation formelle et informelle. À partir d'un dialogue avec lui-même, l'instructeur conscientise ainsi son activité en la verbalisant. En lui rendant sa propre expérience " étrangère ", l'activité peut changer de sens en se réalisant dans des significations nouvelles (Clot, 1999).

La méthode de l'auto-confrontation simple et croisée s'inspire de l'approche du développement de Vygotski (1928) (le rôle du langage et des interactions sociales dans le développement). Le dispositif doit favoriser l'apprentissage par l'intermédiaire de l'influence des autres. Cette démarche de formalisation de l'expérience professionnelle comprend un groupe d'analyse, les enregistrements vidéo permettant la production de séquences filmées associées à la réalisation d'auto-confrontations simples et croisées également filmées, et le retour au collectif de ce travail de co-analyse de l'activité (Clot, Faïta, Fernandez, \& Scheller, 2000). Le dispositif permet alors de mieux cerner la complexité du travail du sujet, les raisonnements cognitifs qui accompagnent ses 
décisions et ses gestes qui sont contraints par la situation. Le sens de l'action mis ainsi en mots produit un effet sur autrui et sur le sujet lui-même, ouvre de nouveaux espaces de développement. Les participants effectuent alors un cycle entre ce qu'ils font, ce qu'ils en disent et ce qu'ils font de ce qu'ils disent (Ibid.). Ils parviennent ainsi à se distancier du genre professionnel grâce à une prise de conscience du style de leurs actions pour finalement développer le métier. En montrant, par ce dialogue, ce qui est fait, mais également ce qui n'a pas été fait, ce qui aurait pu être fait; cela contribue à faire remonter les dilemmes de l'activité et les potentialités non exprimées dans l'activité réalisée. Simuler ce qui n'est pas en train de se faire pour convoquer ce qui pourrait l'être. C'est dans l'exploration des autres solutions possibles que se joue la simulation. Ici, la simulation est un vecteur possible de la création.

Enfin, l'objet technique peut aussi être utilisé comme une méthode d'analyse du travail et d'élaboration de l'expérience, par la simulation. Elle est issue des courants de la pédagogie inductive et de l'entraînement mental. Élaborée à son origine par Simondon (1958), philosophe, cette méthode a été reprise par Marcon (1976) qui l'a adaptée dans le champ de la formation des adultes. Ainsi, la méthode de l'objet technique propose une approche où un collectif de travail est réuni autour d'un objet du travail. L'objet technique a alors trois fonctions: analyseur de situations de travail par l'effort de formalisation de l'objet et la façon dont il est utilisé; médiateur de processus de coopération; et révélateur de l'expérience et de la créativité de l'individu. L'objectif est de partir de l'objet, de le déconstruire pour le reconstruire afin de tirer sa loi de fonctionnement, pour le transformer et ainsi se transformer (Moncorger, \& Gaubert, 2013). Par exemple, à partir d'un objet technique pris dans l'activité, la méthode permet de simuler et d'expérimenter une situation de formation "de l'objet à la loi» (Gaubert, 2012), par l'apprentissage d'une méthode inductive transférable sur d'autres situations. Vecteur de professionnalisation, l'objet technique permet de faire remonter à la surface ce qui est engagé dans le travail et de rendre visible l'ingéniosité du professionnel.

31 En définitive, ces différentes méthodes semblent être des outils de simulation à visée réflexive et constructive en tant qu'elles participent à la réélaboration des expériences et des connaissances pour de nouvelles expériences. Elles permettent de revivre, par ces moyens détournés de simulation, l'expérience pour la comprendre et transformer les fonctionnements réalisés en objet de nouveau fonctionnement. C'est une prise de conscience du sujet qui entre dans des dialogues intérieurs et extérieurs. Cette simulation participe aussi d'un mouvement de professionnalisation qui peut s'initier à partir des situations professionnelles. Assez paradoxalement, on peut aussi dire qu'elle contribue au développement d'une position pro-active, qui va faciliter l'adaptation à des situations futures à partir de l'analyse du présent.

\subsection{Simulation projective}

32 La simulation projective vise à l'aide de différents outils à faire rejouer à un individu et/ ou un collectif une situation éprouvante d'un point de vue subjectif et psychique (persistance des émotions ressenties) passé ou à venir. La simulation devient alors un " miroir » déformant le réel, pouvant mettre en évidence le contenu subjectif de la situation de référence. L'usage de ce type de méthodes favorise les processus associatifs des acteurs, établis spontanément en leur permettant d'exprimer des attitudes, motivations implicites, non forcément conscientes. Les utilisateurs peuvent ainsi exprimer tout ce 
qu'ils refusent d'être, ce qu'ils ressentent, comme mauvais ou comme étant leurs points vulnérables. À ce titre, ces méthodes aident les acteurs «à réfléchir, à entrer en euxmêmes, à renouer avec des aspects méconnus d'eux même tout en facilitant la prise de parole et l'expression personnelle» (Bélisle, 2014, p. 17). Autrement dit, ces méthodes aident les participants, individuellement et collectivement, à mettre en mots et à expliciter leurs représentations et émotions sur un thème ou un objet. Plusieurs méthodes peuvent servir cette perspective particulière de la simulation.

Le debriefing regroupe un ensemble de pratiques qui vise à traiter des personnes ayant vécu un événement stressant ou potentiellement traumatique. Dans les contextes professionnels, il est généralement une réponse que les organisations mettent en place dans le cadre de l'intervention sur les violences au travail. Il aide à la prise en charge post-traumatique des victimes et de l'entourage professionnel. Le debriefing collectif est une technique permettant l'expression des salariés concernés par l'événement et se réalise dans un temps proche de l'événement (Rouat, \& Laporte, 2011). Le travail de simulation réside ici dans le fait de se replonger ensemble dans la scène qui a fait effraction en travaillant deux niveaux d'élaboration : l'un centré sur les faits permettant de reconstituer collectivement le déroulement de l'acte traumatisant et éviter de laisser des vides laissant place à l'angoisse ; l'autre centré sur les émotions dans le but d'atténuer les éprouvés et les symptômes. Le fait de parcourir minutieusement l'événement, telle une investigation, au niveau des faits, des émotions et des pensées, pour retrouver ce qui l'a sidéré à cet endroit, participe à ce travail thérapeutique permettant de se libérer ou d'intégrer l'expérience traumatique. En facilitant alors la construction du lien, ce qui était disjoint se rejoint.

La méthode du théâtre forum (Guerre, 1999) a pour objectif de soutenir des communautés ou des groupes rencontrant des difficultés par le biais de l'échange collectif et du jeu. Cette méthode permet de développer la capacité des individus à interagir collectivement au sein d'un groupe, mais aussi à mieux évaluer des situations de conflits et/ou des situations stressantes (Grosjean, \& Morand, 2018). Synthétiquement, cette méthode se décompose en trois temps. Tout d'abord, les acteurs jouent une histoire réelle de conflit vécu par le public concerné et cela termine par un temps d'échange entre spectateurs qu'on appelle temps de "forum » où les spectateurs évoquent leurs émotions et leurs ressentis vis-à-vis de la scène. Le Joker/animateur propose alors à un ou plusieurs spectateurs de remplacer un ou plusieurs acteurs et d'endosser leurs personnages. Les spectateurs deviennent ainsi Spect-Acteurs (Guerre, 1999). Ils participent alors à la scène et donnent à l'histoire un autre tournant.

La méthode du psychodrame (Moreno, cité par Martin, 2015) a pour objectif thérapeutique de libérer les émotions des individus qui ne s'expriment pas du fait de rôles sociaux rigides. Cette méthodologie constitue, selon Moreno (op.cit.), un " apprentissage de la spontanéité " pour les individus par le biais d'une transmission «de nouvelles combinaisons d'actes et de transformation" face à une situation nouvelle. Synthétiquement, le psychodrame se déroule lors d'une séance en groupe (avec deux animateurs et des participants) au cours de laquelle, les participants commencent par évoquer des souvenirs, rêves, sensations. Ils mettent en scène cette situation avec l'aide du groupe en choisissant d'attribuer des rôles à des membres du groupe. Ils jouent ensuite la scène puis des échanges s'organisent autour de la scène qui a été jouée. L'animateur qui ne joue pas doit alors veiller à favoriser l'expression du vécu de chaque personne tout en veillant à faire respecter le cadre déontologique et méthodologique 
(Cru, 2005). Cette approche se différencie du théâtre forum dans la mesure où le formateur cherche à travailler les raisons des blocages ou des représentations inadéquates face à une situation donnée.

La méthode des incidents critiques a été développée à l'origine par Flanagan (1954) dans le but de résoudre des problèmes pratiques liés à l'activité d'un individu, par un retour réflexif et critique sur des expériences positives ou négatives passées. Elle vise à faire expliciter, par une démarche introspective, les situations, les conditions, les circonstances et les contingences (sociales, artéfactuelles, organisationnelles, situationnelles ou encore dispositionnelles) ayant contribué à générer une situation dégradée ou au contraire bénéfique du point de vue du sujet. Butterfield \& Al. (2005 cité par Leclerc, Bourassa, \& Filteau, 2010) ont mis en évidence le fait que l'utilisation de cette méthode s'est transformée au fil du temps. Elle est ainsi utilisée pour mieux comprendre les construits psychosociaux contenus dans l'expérience subjective et intersubjective des individus. Pour ces raisons, il est donc possible de rattacher cette méthode groupale des incidents critiques à la simulation émotionnelle projective alors que son projet originel, comme évoqué plus haut, s'attachait plutôt à la simulation réflexive. Synthétiquement, cette orientation prend la forme d'une séance de groupe, durant laquelle un individu aborde un évènement marquant pour lui, généralement problématique, dans son exercice professionnel. Il explique le contexte de l'incident, puis décrit la séquence en relatant les faits et gestes de la situation. Le groupe effectue ensuite un retour sur les interprétations possibles de l'incident en s'interrogeant sur les intentions, les intérêts des acteurs et les émotions ressenties. Un temps d'incubation est ensuite proposé au groupe afin de prendre du recul. Enfin, le groupe se réunit à nouveau pour un temps de retour réflexif au cours duquel les membres du groupe échangent sur leurs interprétations respectives. Selon Leclerc, Bourassa et Filteau (2010, p. 27), « ce qui est en jeu, c'est bien plus que les méthodes de travail ou les gestes professionnels : c'est l'identité de la personne, ses peurs, ses déceptions, ses espoirs, son rapport aux autres et à elle-même, voire le sens même de son existence ». Et c'est justement en cela que cette méthode relève d'une phase de simulation où l'éprouvé subjectif de la personne est convoqué : la personne déroule et expose son activité à l'attention des autres et, se faisant, à sa propre attention. Si le sujet ne simule pas explicitement et directement l'activité (au sens de feindre), elle la rejoue cependant intérieurement pour être en capacité de la formuler. Elle se voit refaire les actions et ce faisant, ressent les choses de ce passé. C'est cette expérimentation subjective et intériorisée qui se rapproche dès lors de la simulation projective.

Enfin, la méthode des cartes projectives et/ou du photo langage consiste à présenter une série d'images (photos, dessins, photomontages...) à des utilisateurs en leur demandant de choisir celles qui caractérisaient le plus leurs ressentis et représentations vis-à-vis de la situation qu'ils viennent de vivre (un changement technologique, organisationnel par exemple) (Anzieu, \& Chabert, 2004; Laneyrie, Rouat, \& Bobillier Chaumon, 2018). Cette méthode permet aux utilisateurs de construire a posteriori la signification de leur vécu en attribuant des détails, des propriétés aux cartes choisies. La composante psychique du sujet en activité est alors convoquée dans la mesure où l'on demande à ce dernier d'expliciter l'émotion, l'éprouvé subjectif auquel la carte sélectionnée lui parait associée. L'artefact graphique devient donc un médiateur, un facilitateur pour l'élaboration de son expérience tout à la fois singulière et subjective. Il donne aussi des repères et des jalons pour (se) rejouer la situation et ainsi mieux " comprendre des comportements complexes, ou pratiquer une modélisation d'un savoir-faire, [pour] 
permettre d'accéder aux procédures et processus cognitifs qui sous-tendent ces comportements. La méthode peut aussi servir à identifier les raisons d'agir, les buts recherchés et les motivations qui guident l'action » (Brangier, Lancry, \& Louche, 2006, p. 24).

Au final, il apparait que ces méthodes de simulation projective permettent à l'individu à partir de l'identification d'un changement externe (situation problématique, changement organisationnel, technique, social) de projeter des objets internes (affects, représentations) dans le but de transformer leur manière d'appréhender la situation future. À ce titre, ces méthodes interrogent le travail vivant, ressenti. L'objectif est de proposer un temps individuel et/ou collectif aux individus afin qu'ils puissent penser leur activité (Daniellou, 1988) et trouver ainsi des solutions ingénieuses pour faire face aux difficultés rencontrées.

\subsection{Simulation rétrospective}

39 La démarche de simulation rétrospective porte sur l'activité « qui s'est faite ». Elle permet de faire revivre/rejouer une situation professionnelle terminée depuis un laps de temps s'échelonnant de quelques mois à plusieurs années afin de pouvoir en saisir le vécu et la perception de l'acteur. Cette expérience vécue peut se définir comme « les flux d'actions, émotions, pensées et sensations perceptives qui émergent dans le cours de l'activité et dont le sujet est, ou peut être rendu réflexivement conscient via des méthodes idoines » (Cahour, Salembier, \& Zouinar, 2016, p. 259). Cette méthode de simulation a pour but de recueillir, de formaliser et de modéliser cette expérience vécue (sociale, émotionnelle, personnelle, professionnelle...) pour concevoir et reconcevoir des situations ou pour réajuster des dynamiques de travail (collectives, interpersonnelles...) (Cacheux, Bobillier Chaumon, \& Cuvillier, 2018). Dans les méthodes rétrospectives, on compte deux mouvements : le premier ramène sur l'activité passée; le second réouvre vers le champ des possibles pour les situations à venir. En cela, elles constituent des situations de simulations. Revisiter certaines situations c'est se donner la possibilité d'en saisir une autre compréhension, susceptible d'enrichir les possibilités d'action future. Le processus d'évocation permet l'accès à ce qui s'est réalisé, mais fait apparaître ce qui n'a pu se faire, ouvrant les autres possibles de l'activité. Les méthodes mobilisées peuvent ainsi être détournées partiellement, pour non pas spécifiquement accéder à l'activité réalisée, mais au réel de l'activité. Nous pouvons à titre d'exemple, citer quelques méthodes qui peuvent être utilisées dans le cadre d'une simulation rétrospective :

- Le REX (Retour d'EXpérience), dispositif associé aux métiers de la sécurité, "vise à maîtriser les perturbations futures par une activité réflexive sur les événements passés » (Gaillard, 2005). Cette démarche permet de comprendre et corriger des défaillances (techniques, humaines ou organisationnelles) en permettant de développer des connaissances de l'action. Elle s'attache non seulement à produire une compréhension des événements, mais également une mise en commun des connaissances, pour améliorer la sécurité des systèmes. Différentes versions du REX sont proposées dont le choix d'utilisation varie selon les secteurs de production et la décision managériale. Sans être exhaustif, on recense des REX sur les accidents, dans les systèmes à hauts risques (fiabilité des systèmes, sécurité des procédés), réactif (rétrospectif en analysant les évènements passés), prospectif (en anticipant le futur), événementiel (simple retour sur événement), classique (retour sur les écarts à la norme), probabiliste (à partir des données collectées 
vise à la performance de l'outil industriel, la continuité des services dans le cadre des transports).

41 Nous avons fait le choix de faire figurer l'entretien d'explicitation (Vermersch, 1994) en le détournant de sa visée originelle. Il s'agit de favoriser la conscientisation des souvenirs d'une action passée, pour faciliter l'émergence d'autres possibles. Cette approche vise dans un premier temps à obtenir une description aussi fine que possible d'une activité passée, réalisée par une personne en situation de pratique professionnelle ou engagée dans la réalisation d'une tâche. L'entretien d'explicitation permet de guider le sujet dans la verbalisation précise du déroulement de son activité. Elle relève d'une activité de mise en mots de "qu'est-ce qui a fait que » en guidant le sujet dans la verbalisation précise du déroulement de sa conduite. Cet entretien facilite l'accès à des dimensions du vécu de l'action qui ne sont pas immédiatement présentes à la conscience de la personne. Dans le cadre d'une simulation rétrospective, le recours à ce type d'entretien permet de relier émotions et conduites sociales de personnes durant une période du changement dans les organisations. Elle aide ensuite à faire ressortir les conflits de critères qui participent à la prise de décision en situation, tout en questionnant la pertinence de plusieurs alternatives.

42 - L'Analyse des Pratiques Professionnelles (ou de la pratique professionnelle) (APP), qui recouvre des pratiques d'intervention différentes, vise essentiellement la professionnalisation et ainsi l'évolution de l'identité professionnelle (Fablet, 2002). Elle contribue à renforcer les compétences et à l'élaboration des enjeux du travail grâce à la mise en discours de l'agir professionnel. Elle accompagne alors les évolutions du travail pour les professionnels qui y sont engagés. Pratiquement, les professionnels réunis en groupes de pairs échangent autour de situations de travail problématiques vécues. Ces rencontres animées par un tiers sont proposées dans un espace sécurisé (règles précises de fonctionnement du groupe) garantissant l'expression des participants. L'APP contribue au développement des ressources collectives pour mieux faire face aux dilemmes auxquels les professionnels sont convoqués dans l'activité. L'APP est particulièrement présente dans les secteurs sociaux, médico-sociaux et sanitaires. Ils s'apparentent à ce qui a tendance à se répandre dans les organisations, c'est-à-dire aux espaces de discussion sur le travail (Detchessahar, 2013).

Quelles sont les limites de la simulation rétrospective? Elles sont de deux ordres :

- D'une part, cette mise en scène de l'activité vécue peut parfois être éloignée du réel. Elle est une reconstruction forcément subjective d'une situation qui rend compte d'une activité vivante (c'est-à-dire ressentie, éprouvée) et non des conditions réelles. Ce biais n'est toutefois pas inhérent à cette méthodologie, mais plutôt au fait d'étudier une situation passée pouvant se dérouler dans certains cas sur une longue durée ;

- D'autre part, les capacités cognitives propres du sujet limitent la restitution de ces expériences passées, incluant divers biais socio-cognitifs (d'attribution, de confirmation, d'autocomplaisance, de négativité) inhérents à cet exercice.

Ces altérations possibles peuvent compromettre la réalité et la véracité des propos relatés. Pour autant, n'est-ce pas justement le ressenti de ce changement qui doit être finement appréhendé? 


\section{Discussion et conclusion}

$\mathrm{Au}$ terme de ce travail de formalisation des approches et techniques de simulation, nous souhaitons discuter certains points importants de controverses mis en avant par les auteurs.

\subsection{Caractéristiques des simulations présentées}

Nous retiendrons d'abord que la simulation permet de remettre en scène des situations de travail pour leur donner un autre statut, d'autres possibles et faire en sorte que le réel ne se confonde pas avec le réalisé. En tentant d'articuler ce qui s'est fait, ce qui se fait, ce qui se fera, ce qui ne se fait pas, la simulation contribue à développer chez les personnes des "pouvoirs faire ", ou plutôt des "possibilités de faire ». Le tableau 1 ci-dessous propose de synthétiser les différents apports de chacune des simulations analysées.

Tableau 1. Caractéristiques de chacune des simulations présentées.

Table 1. Characteristics of each presented simulation

\begin{tabular}{|c|c|c|c|c|}
\hline $\begin{array}{l}\text { Types de } \\
\text { simulation }\end{array}$ & $\begin{array}{l}\text { Prospective/ } \\
\text { prédictive }\end{array}$ & $\begin{array}{l}\text { Réflexive/ } \\
\text { constructive }\end{array}$ & Projective & Rétrospective \\
\hline Temporalité & Activité à venir & $\begin{array}{l}\text { Activité en } \\
\text { devenir }\end{array}$ & $\begin{array}{l}\text { Activité vivante, } \\
\text { persistante }\end{array}$ & Activité terminée \\
\hline Méthodes & $\begin{array}{lr}\text { MES, Serious } & \text { game, } \\
\text { jeu de rôles, } & \text { Living } \\
\text { simulation } & \\
\text { informatique }\end{array}$ & $\begin{array}{l}\text { Auto- } \\
\text { confrontation } \\
\text { simple et } \\
\text { croisée, } \\
\text { instruction au } \\
\text { sosie, objet } \\
\text { technique }\end{array}$ & $\begin{array}{l}\text { Psychodrame, } \\
\text { débriefing, } \\
\text { photolangage, cartes } \\
\text { projectives, théâtre } \\
\text { forum, Incidents } \\
\text { critiques }\end{array}$ & $\begin{array}{l}\text { Analyse des } \\
\text { pratiques } \\
\text { professionnelles, } \\
\text { REX, entretien } \\
\text { d'explicitation }\end{array}$ \\
\hline & \multicolumn{4}{|c|}{$\begin{array}{l}\text { Pour comprendre et transformer } \\
\text { les individus, le collectif et/ou la situation de travail et/ou le métier et/ou le } \\
\text { projet }\end{array}$} \\
\hline
\end{tabular}

Visées de la

simulation 


\begin{tabular}{|c|c|c|c|c|}
\hline & $\begin{array}{lrr}\text { Créer } & \text { un } & \text { espace } \\
\text { d'élaboration } & \\
\text { collectif } & \\
\text { permettant } & \\
\text { d'anticiper } & \text { les } \\
\text { enjeux } & \text { d'une } \\
\text { situation à } & \text { venir } \\
\text { et } & \text { maîtriser } & \text { les } \\
\text { incidences } & \\
\text { potentielles } & \text { sur } \\
\text { les individus, } & \text { les } \\
\text { collectifs } & \text { et } & \text { les } \\
\text { organisations. } & \\
\text { L'objectif } & \text { est } & \text { de } \\
\text { coadapter } & \text { et } \\
\text { transformer } & \text { le } \\
\text { milieu } & \text { et } \\
\text { l'individu } & \\
\text { Anticiper } & \text { pour } \\
\text { transformer } & \end{array}$ & 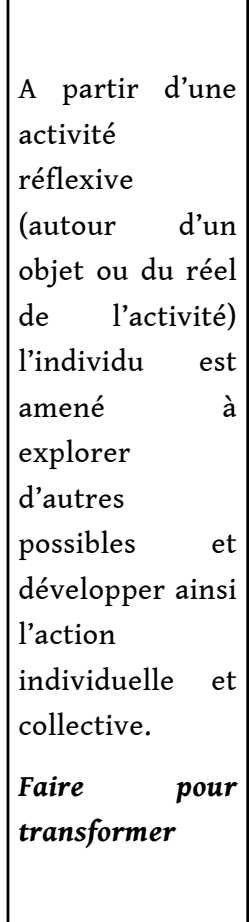 & $\begin{array}{l}\text { A partir de } \\
\text { l'identification d'un } \\
\text { objet externe } \\
\text { (situation } \\
\text { problématique, } \\
\text { changement, } \\
\text { organisationnel, } \\
\text { technique, social), } \\
\text { l'individu est amené } \\
\text { à projeter des objets } \\
\text { internes (affects, } \\
\text { représentations) } \\
\text { afin de transformer } \\
\text { la situation future } \\
\text { ou de se transformer } \\
\text { lui-même. } \\
\text { Se projeter pour } \\
\text { transformer }\end{array}$ & $\begin{array}{l}\text { A partir d'une } \\
\text { activité réflexive } \\
\text { sur une situation } \\
\text { réelle et passée, } \\
\text { l'individu est amené } \\
\text { à agir sur les aspects } \\
\text { individuels la } \\
\text { collectifs dans le but } \\
\text { de renforcer la } \\
\text { compréhension des } \\
\text { enjeux des } \\
\text { situations de travail } \\
\text { et in fine les } \\
\text { transformer. } \\
\text { Reconvoquer pour } \\
\text { transformer }\end{array}$ \\
\hline $\begin{array}{l}\text { Destinataires } \\
\text { potentiels }\end{array}$ & \multicolumn{4}{|c|}{ Opérateur, collectif de travail ; préventeurs, partenaires } \\
\hline $\begin{array}{l}\text { Concepts } \\
\text { utilisés }\end{array}$ & \multicolumn{4}{|c|}{$\begin{array}{l}\text { Activité, réel de l'activité, genre professionnel, style, métier, pouvoir d'agir, créativité, } \\
\text { collectif de travail }\end{array}$} \\
\hline
\end{tabular}

Ce tableau montre que l'usage de la simulation lors d'une intervention nécessite de prendre en compte plusieurs dimensions : la temporalité de la simulation, les méthodes associées, la visée de la simulation, les destinataires potentiels et enfin les concepts associés. Il montre également la diversité des visées de la simulation qui peuvent être plus ou moins complémentaires et/ou conflictuelles : une visée adaptative (adapter l'individu à son environnement; adapter l'environnement à l'individu) qui donne lieu à des modifications environnementales (matérielles, sociales, réglementaires...) et/ou à des modifications individuelles (comportements, perceptions, croyances, efficiences, émotions...) qui ne sont pas toujours compatibles, par exemple, avec une visée transformatrice de l'activité. Cette dernière s'appuie sur le développement des potentialités individuelles et collectives et se construit dans l'action collective, déontique, transgressive. Enfin, on peut relever que l'une des visées transversales de la simulation, instaurée dans les milieux professionnels, est celle de la professionnalisation.

\subsection{Des simulations qui s'imbriquent au sein d'une perspective temporelle dynamique}

Il faut souligner ensuite la perspective dynamique qui existe à la fois entre les approches et au sein d'une même approche. Si cette taxinomie aide à clarifier le rôle des différentes formes de simulation dans le développement de l'action ainsi que leurs visées, nous n'avons pas voulu faire une compilation des techniques de simulation, car celles-ci ne 
sont pas cloisonnées. Elles s'imbriquent, se chevauchent, parfois se confrontent. Il existe donc une certaine plasticité des méthodes susceptibles d'être mobilisées dans la simulation, selon les usages que l'on en a. Par exemple, la méthode « serious game » dont la finalité est le développement de nouvelles ressources pour l'individu par une simulation virtuelle d'une activité a été rangée dans la rubrique "Simulation prospective». Pour autant, l'élaboration d'une réflexion sur ses pratiques à partir d'une confrontation à des scénarii d'actions possibles semble aussi proche des orientations de types «Simulations constructives». Dans ce cas, la proximité paraît en effet grande entre d'une part, viser le développement de nouvelles ressources pour l'individu et d'autre part, s'interroger sur l'expérience et le métier dans le cadre d'une démarche développementale orientée sur le pouvoir d'agir. La différence semble donc tenue au regard d'un objectif de transformation qui vise l'individu et ses connaissances pour l'action.

De plus, à l'intérieur d'une même approche, on peut repérer des dynamiques temporelles divergentes. Par exemple, dans la simulation projective, on peut mettre au travail une expérience émotionnelle passée qui reste éprouvante dans le présent et sur laquelle on voudra agir pour qu'elle ait un sens nouveau à l'avenir. De même, on peut travailler, dans cette même approche, l'émotion suscitée par l'annonce d'un changement organisationnel particulièrement anxiogène, pour mieux l'accompagner. L'approche projective contient également une dimension anticipatrice et historique, que nous retrouvons dans l'approche réflexive. Enfin, la confrontation à des situations supportables (d'intensité affective supportable) permet d'aborder mentalement des situations plus "anxiogènes ». Par ailleurs, nous savons que l'activité, qu'elle soit actuelle ou à venir s'inscrit toujours dans une histoire plus grande, un passé qui agit encore, dans un patrimoine commun, culturel et collectif. Un autre exemple porte sur la méthode de l'étude de cas, qui fait l'objet de multiples usages dans les sciences humaines et sociales. En effet, l'usage de l'étude de cas interroge les frontières temporelles du passé, présent, futur. Pour Leplat (2002, p. 1), «un cas est un objet, un événement, une situation constituant une unité d'analyse [...]. Celle-ci est découpée dans la réalité et prend forme dans un contexte particulier qui pèse sur elle. Le cas a une épaisseur temporelle plus ou moins grande dans la mesure où il peut s'agir d'une situation considérée à un moment donné ou d'une situation dans son développement ». Le cas auquel nous nous référons peut permettre de mieux comprendre une situation passée pour agir dans l'ici et maintenant, voire dans l'ailleurs et plus tard. Il peut également découler d'une scénarisation, qui explicitement incite à se projeter dans un futur plus ou moins proche, pour tenter de faire évoluer une activité. La puissance évocatrice de ce scénario doit contribuer à développer un imaginaire qui enrichit le réel auquel s'adosse le concepteur de l'étude de cas. L'enjeu dans la simulation, est d'introduire du jeu pour contribuer à ouvrir des espaces insoupçonnés à une réalité dont nous avons difficulté à nous extraire. L'intervenant utilisant les méthodes de simulation, participe à rendre poreuses les frontières entre les types de simulation.

\subsection{Des simulations ayant une plasticité importante permettant de sortir d'un cadre temporel strict}

Les différentes méthodes de simulation peuvent, par ailleurs, être utilisées par les acteurs, du fait de leur plasticité, hors de leur strict cadre temporel. On observe en effet une richesse dans la mixité méthodologique mobilisable dans ces formes de simulations 
qui peuvent emprunter une diversité d'outils. Elle réclame expérience et rigueur pour l'intervenant qui l'utilise et constitue une ressource pour développer son action propre. Cette activité d'appropriation donne lieu au développement de «styles" de simulation chez l'intervenant, qui sortent, débordent des types de simulation : cette réflexion nous entraine sur ce terrain bien connu des écarts qui sont comblés, pour que "ça marche ", par une activité (prescrit/réel ; procédures/processus ; connaissance/action...). De plus, celui-ci possède une liberté de déformer l'outil, de l'adapter aux contraintes du terrain, et d'adapter l'intervention aux limites des méthodes elles-mêmes, qui représentent des contraintes diverses dans leur mise en œuvre. Il peut s'agir de leurs coûts (financier, implication des acteurs, temps consacré, action pluri-partenaires...), et des implications méthodologiques (par exemple, le déploiement matériel et technique exigé, le dévoilement de soi dans la simulation, la centration sur l'activité qui peut se heurter à bon nombre de résistances organisationnelles qui facilitent des approches centrées sur l'individu...). Compte tenu de ces exigences, la simulation requiert donc une négociation et une acceptation préalable des différentes parties prenantes du projet afin d'être en mesure de pouvoir instruire convenablement la démarche dans le processus d'intervention. Ce mouvement d'appropriation/transformation porté par les utilisateurs contribue à développer une simulation qui peut se démarquer d'une approche par trop modélisante.

\subsection{Perspectives et limites de la simulation}

51 Cet exercice nous pousse à interroger autrement la visée transformatrice des méthodes qui sont engagées sur le terrain. Qu'est-ce qu'on cherche à transformer? Est-ce le milieu, le projet, l'individu? Est-ce qu'on cherche à éviter des situations problématiques? A développer des ressources? Nous pouvons constater que la plupart de ces techniques agissent en effet sur un niveau individu et/ou collectif. Nous pouvons, par conséquent, nous poser la question de leur capacité à agir sur l'environnement de travail.

De plus, ces questions exigent de penser la technique de simulation comme une étape d'un processus de transformation plus globale, et non comme un état. Penser la simulation dans un cadre d'intervention plus large c'est instaurer les conditions d'une inscription durable de l'action, c'est permettre aux acteurs de l'organisation de se saisir de ce processus pour qu'il continue d'exister après la simulation en développant leur action. En ce sens, la simulation peut devenir «simul-action». Il est particulièrement dommageable pour les individus de se retrouver, parfois par la contrainte, dans une activité de simulation, alors qu'autour d'eux, le questionnement sur le travail sur son sens ne se développe pas. Se poser la question de savoir ce qu'il reste après une simulation permet de redonner toute sa place à ceux qui ont à développer ce dialogue.

Enfin, cette réflexion nous amène encore à penser aux destinataires de ces simulations. À qui sont destinés les développements, les apports de ces simulations? Cette question $a$ priori évidente ne l'est pas pour autant. D'une part parce qu'il peut exister plusieurs destinataires, directs et indirects (les partenaires); d'autre part parce que parfois visée et destinataires peuvent ne pas se rejoindre. Si nous avons choisi dans ce papier d'évoquer les bénéfices susceptibles d'être tirés des méthodes de simulation, cela n'évacue pas pour autant les quelques limites qu'il faut souligner. Dans quelle mesure, la simulation retranscrit-elle bien le réel ? Cette question peut se poser à différents niveaux : soit dans ce que le sujet dit ou ne dit pas de son activité (simulation rétrospective ou projective); 
soit dans ce qui se passe au cours de la simulation entre les protagonistes (simulation réflexive) ou du rôle de l'intervenant qui va fixer un cadre de simulation (scénario de la simulation prospective). De quelles(s) réalité(s) ce qui est mis en scène relève-t-il ? Nous soulevons ici les bénéfices à tirer de la simulation, sans oublier que, dans son sens premier, la simulation désigne une dissimulation, un mensonge.

\section{BIBLIOGRAPHIE}

Anzieu, D., \& Chabert C. (2004). Les méthodes projectives. Paris : PUF.

Baccino, T., Bellino, C., \& Colombi, T. (2005). Mesure de l'utilisabilité des interfaces. Paris : Hermès.

Barcellini, F., Van Belleghem, L., \& Daniellou, F. (2013). Les projets de conception comme opportunité de développement des activités. In Falzon, P. (coord.) Ergonomie constructive (pp. 191-206). Paris : PUF.

Baudrillard, J. (1981). Simulacres et simulation. Paris : Editions Galilée. 240 p.

Belisle, C. (2014). Le photolangage : Communiquer en groupe avec des photographies : présentation de la méthode. Paris : Broché.

Bobillier Chaumon, M.E. (2016). Acceptation située des TIC dans et par l'activité : Premiers étayages pour une clinique de l'usage. Psychologie du Travail et des Organisations, 22(1), 4-21.

Bobillier Chaumon, M.E., \& Clot, Y. (2016). Clinique de l'usage. Les artefacts technologiques comme développement de l'activité. Activités, 13(2), 1-7. Consulté le 5/12/2017 sur http:// activites.revues.org/2897; DOI : 10.4000/activites.2897.

Brangier, E., Lancry, A., \& Louche, C. (2006). Les dimensions humaines du travail. Théories et pratiques en psychologie du travail et des organisations, Nancy : Presses universitaires de Nancy.

Cacheux, M., Bobillier Chaumon, M.E., \& Cuvillier, B. (2018). La Simulation Rétrospective : une approche des comportements émotionnels et sociaux vécus au cours d'un changement professionnel. In Actes du Congrès international de Psychologie du travail AIPTLF 2018. Bordeaux, 9-12 juillet 2018. (À paraître).

Cahour, B., Salembier, P., \& Zouinar, M. (2016). Analyzing lived experience of activity. Le Travail humain, 79, (3), 259-284.

Clot, Y. (1999). La fonction psychologique du travail. Paris : PUF.

Clot, Y. (2008). Travail et pouvoir d'agir. Paris : PUF.

Clot, Y., \& Faïta, D. (2000). Genre et style en analyse du travail. Concepts et méthodes. Travailler, 4, 7-42.

Clot, Y., Faïta, D., Fernandez, G., \& Scheller, L. (2000). Entretiens en auto-confrontation croisée : une méthode en clinique de l'activité. Pistes, 2(1), 1-7.

Cru, D. (2005). Groupe en psychodrame, groupe en entreprise : un jalonnement. Psychodrame, la revue du psychodrame freudien. $145,72-80$. 
Daniellou, F. (1988). Ergonomie et démarche de conception dans les industries de processus continus. Quelques étapes clés. Le Travail humain, 51(2), 185-194.

Daniellou, F. (2007). Des fonctions de la simulation des situations de travail en ergonomie. Activités, 4(4-2), 77-83.

Detchessahar, M. (2013). Faire face aux risques psycho-sociaux : quelques éléments d'un management par la discussion. Négociations, 1(19), 57-80.

De Visscher, P. (1979). Des exercices structurés en dynamique des groupes : pourquoi ? Les Cahiers de Psychologie Sociale, 2, 1-4.

Fablet, D. (2002). Travail social et analyse des pratiques professionnelles : les éducateurs et leurs modèles de référence. Recherche et formation, 39, 51-68.

Flanagan, J.C. (1954). The Critical Incident Technique. Psychological Bulletin, 51(4),327-58.

Gaillard, I. (2005). Facteurs socio-culturels de réussite du REX industriel par l'analyse bibliographique. Cahiers de la Sécurité Industrielle, 2008-01, consulté le 10/01/2018 sur http:// www.icsi-eu.org/francais/dev_cs/cahiers/

Gaubert, T. (2012). Activités et développement. In Bobillier Chaumon, M.-E. \& Sarnin, P. (Eds) Manuel de Psychologie du Travail et des organisations. (pp. 95-115). Bruxelles : De Boeck.

Grosjean, V., \& Morand, O. (2018). Analyse du travail, construction et transformation du réel. Appréhender les conflits par le théâtre-forum et l'approche systémique. Actes du $53^{\text {ème }}$ Congrès de la SELF, Bordeaux, 3-5 Octobre 2018 (à paraître).

Guerre, Y. (1999). Le théâtre forum, pour une pédagogie de la citoyenneté. Agora débats/jeunesses, 17(1), p. 142.

Guibourdenche, J., Salembier, P., Poizat G., Haradji, Y., \& Galbat, M. (2015). A Contextual Approach to Home Energy Management Systems Automation in Daily Practices. Proceedings of European Conference on Cognitive Ergonomics (ECCE 2015), 1 - 3 July 2015, Warsaw, Poland. ACM.

Guibourdenche, J., Vacherand-Revel, J., Fréjus, M., \& Haradji, Y. (2015). Analyse de contextes d'activité domestique pour la conception de systèmes diffus énergétiquement efficients. Activités ,12(1), 46-69. Consulté le 5/02/2018 ; DOI : 10.4000/activites.994

Kent (de) T. (2003). Le roman d'Alexandre ou le roman de toute chevalerie. Traduction, présentation et notes de Catherine Gaullier-Bougassas et Laurence Harf-Lancner, avec le texte édité par Brian Foster et Ian Short. Paris : Champion.

Kostulski, K., Clot, Y., Litim, M., \& Plateau, S. (2011). L'horizon incertain de la transformation en clinique de l'activité : une intervention dans le champ de l'éducation surveillée. Activités, 8(1), pp. 129-145, Consulté le 5/02/2018 : http://www.activites.org/v8n1/v8n1.pdf

Laneyrie, E., Rouat, S., \& Bobillier Chaumon, M.-E. (2018). Analyser les effets de la complexité des systèmes professionnels mouvants via l'usage de cartes projectives. In Actes du Congrès international de Psychologie du travail AIPTLF 2018. Bordeaux, 9-12 juillet 2018. (A paraître).

Leclerc, C., Bourassa, B., \& Filteau, O. (2010). Utilisation de la méthode des incidents critiques dans une perspective d'explicitation, d'analyse critique et de transformation des pratiques professionnelles. Éducation et francophonie, 38(1), 11-32.

Leplat, J. (2002). De l'étude des cas à l'analyse de l'activité. PISTES, 4(2), 1-31.

Marcon, M. (1976). Réflexion sur l'enseignement technique, Thèse, Université de Lyon 
Martin L., \& Lhuilier D. (2016). Entraînement à la prise de décision avec un serious gaming : délibération et conflits de valeurs. Psychologie du Travail et des Organisations, 22(2), 135-146.

Martin, L. (2015). De l'activité virtuelle à l'activité réelle : ressources et empêchements à la créativité de cadres formés au management d'équipe avec un serious gaming. Thèse de doctorat en Psychologie du Travail, Ecole doctorale Abbé Grégoire, Paris.

Miossec, Y. (2011). Le métier comme instrument de protection contre les risques psychosociaux au travail : le cas d'ingénieurs managers de proximité, Le Travail humain, 4(74), 341-363.

Moncorger, J.-M., \& Gaubert, T. (2013). Créativité, un nouveau regard, théorie et pratique. Paris : L'harmattan.

Pallamin, N., Pavard, B., Zamberlan, M.C., \& Santos, V. (2010). Interactions professionnelles en univers virtuel. In Valléry, G. et al. (Eds). Ergonomie, conception de produits et services médiatisés (57-76). Paris : PUF. DOI : 10.3917/puf.lepo.2010.01.0057

Patin, B. (2005). Le jeu de rôles : pratique de formation pour un public d'adultes. Les Cahiers Internationaux de Psychologie Sociale, 3(67-68), 163-178.

Rouat, S., \& Laporte, J. (2011). Agressions et hold-up. In Grasset, Y., Debout, M., Rouat, S. \& Bachelard, O. Risques psychosociaux au travail : vraies questions et bonnes réponses. (Pp. 133-151). Paris : Editions Liaisons.

Salembier, P., \& Pavard, B. (2004). Analyse et modélisation des activités coopératives situées. Activités, 1(1), pp. 87-99. DOI : 10.4000/activites.1170.

Simondon, G. (1958). Du mode d'existence des objets techniques. Paris : Aubier ; dernière réédition corrigée et augmentée, Paris : Flammarion.

Van Belleghem, L. (2012). Simulation organisationnelle : innovation ergonomique pour innovation sociale. In Dessaigne, M-F., Pueyo, V. \& Béguin, P. Innovation et Travail : Sens et valeurs du changement. Actes du 47ème Congrès de la SELF, 5-7 septembre 2012.

Vermersch, P. (1994), L'entretien d'explicitation en formation continue et initiale, Paris : ESF.

Voilmy, D. (2017). Les Living Lab et la conception participative : l'exemple d'ActivAgeing. Retraite et Société, 75, 125-136.

Vygotski, L.S. (1928/1994). La conception dynamique du caractère de l'enfant. Dans K. Barisnikov \& G. Petitpierre (Eds.), Défectologie et déficience mentale (p. 237-258). Neuchâtel, Paris : Delachaux \& Niestlé.

Vygotski, L.S. (1932/2003). Les émotions et leur développement chez l'enfant, In Conscience, inconscient, émotions (p. 123-153). Paris : La Dispute.

Winnicott, D.W. (1971). Jeu et réalité. Paris : Editions Gallimard. Traduction française 1975.

\section{NOTES}

1. Cela peut prendre des formes très diverses (jeux de rôle, cartes, plateau, sur ordinateur...) 


\section{RÉSUMÉS}

L'objet de ce papier est de proposer une taxonomie fonctionnelle et diachronique des différents types de simulation de l'activité que la journée d'étude, consacrée aux démarches de simulation (organisée par la revue Activités) a permis de mettre en avant. Cette catégorisation repose sur quatre grandes orientations temporelles de la mise en scène de l'activité qui peuvent porter respectivement sur la simulation d'une situation (i) à venir (simulation prospective ou prédictive), (ii) en devenir (constructive ou réflexive), (iii) vivante ou persistante (projective) et (iv) passée (rétrospective). Pour chacune de ces approches, nous exposerons les principales méthodes associées et discuterons de leurs contributions et limites respectives.

The purpose of this paper is to propose a functional and diachronic taxonomy of the different simulation types that the study day, devoted to the simulation process (organized by the Review "Activities"), made it possible to highlight. This categorization is based on four main orientations of the staging of the activity that can relate respectively to the simulation of a situation (i) to come (prospective or predictive simulation), (ii) to become (constructive or reflexive simulation), (iii) alive or persistent (projective simulation) and (iv) past (retrospective simulation). For each of these approaches, we will outline the main associated methods and discuss their respective contributions and limitations.

\section{INDEX}

Mots-clés : simulation, activités, méthodes, approche diachronique, transformation

Keywords : simulation, activities, methods, diachronic approach, transformation

\section{AUTEURS}

\section{MARC-ÉRIC BOBILLIER CHAUMON}

Université de Lyon (Lyon2), Laboratoire GRePS, Université Lyon 2

5 Avenue Pierre Mendès-France, 69676 Bron Cedex

marc-eric.bobillier-chaumon@univ-lyon2.fr

\section{SABRINA ROUAT}

Université de Lyon (Lyon2), Laboratoire GRePS, Université Lyon 2

5 Avenue Pierre Mendès-France, 69676 Bron Cedex

sabrina.rouat@univ-lyon2.fr

\section{ELSA LANEYRIE}

Université de Lyon (Lyon2), Laboratoire GRePS, Université Lyon 2

5 Avenue Pierre Mendès-France, 69676 Bron Cedex

Elsa.Laneyrie@univ-lyon2.fr 


\section{BRUNO CUVILLIER}

Université de Lyon (Lyon2), Laboratoire GRePS, Université Lyon 2

5 Avenue Pierre Mendès-France, 69676 Bron Cedex

bruno.cuvillier@univ-lyon2.fr 